\title{
Courant-Snyder Parameters of Beam Adapters
}

\author{
A. Burov and S. Nagaitsev, FNAL
}

\begin{abstract}
The recently proposed plane-to-vortex beam optical transformation is discussed. The matrix relation for the inverse (vortex-to-plane) transformation is presented. The requirements are formulated in terms of the Courant-Snyder (Twiss) parameters.
\end{abstract}

\section{General Relations}

Recently, Ya. Derbenev has proposed an optical scheme to transform a transverse beam state from flat to magnetized and back [1,2]. This scheme, called by him as the beam adapter, allows to transform a flat beam state into a vortex state, i.e. such that the transverse velocity is perpendicular to the radius, $x x^{\prime}+y y^{\prime}=0$, at any transverse position. If this vortex beam enters an extended solenoid with a properly matched magnetic field, the transverse velocities are canceled inside the solenoid. As a result, the beam becomes magnetized, i. e. its transverse Larmor radius $\rho$ is small compared to the beam size $a$, such that $\rho / a \cong \sqrt{\varepsilon_{y} / \varepsilon_{x}}<<1$, with $\varepsilon_{x}, \varepsilon_{y}$ being the beam emittances, assuming that the entering flat beam was oriented horizontally. This plane-to-vortex transformation is realized by a skew quadrupole block described by a $4 \times 4$ matrix

$$
\mathscr{C}=\mathscr{R}^{-1}\langle\mathrm{M}, \mathrm{N}\rangle \mathscr{R} \text {. }
$$

Here $\mathscr{R}$ is the $45^{\circ}$ rotation, and a symbol $\langle M, N\rangle$ denotes a block-diagonal $4 \times 4$ matrix

$$
\langle M, N\rangle \equiv\left[\begin{array}{cc}
M & 0 \\
0 & N
\end{array}\right] \text {. }
$$

where $\mathrm{M}$ and $\mathrm{N}$ are $2 \times 2$ symplectic matrices. For $2 \times 2$ matrices, the symplecticity reduces to a single requirement on the matrix determinant: $\operatorname{det} M=\operatorname{det} N=1$. The matrix $\mathscr{C}$ can be also expressed as

$$
\mathscr{C}=\frac{1}{2}\left[\begin{array}{ll}
M+N & M-N \\
M-N & M+N
\end{array}\right] .
$$

The block (1) complemented with an adjacent downstream solenoid provides a plane-tomagnetized transformation if the matrices $M$ and $N$ are related to each other as

$$
\mathrm{N}=\mathrm{FM}, \quad \mathrm{F}=\left[\begin{array}{cc}
0 & \beta \\
-1 / \beta & 0
\end{array}\right],
$$

where $\beta$ is the Larmor beta-function in the solenoid with a field $B$ :

$$
\beta=2 p_{0} c /(e B),
$$

$p_{0}$ is the total longitudinal momentum, $-e$ is the electron charge and $c$ is the velocity of light. The matrix equation (3) imposes three independent conditions on matrix elements. Any skew quadrupole block (1) satisfying the conditions (3) transforms a flat, 
horizontally or vertically oriented, beam state into a vortex state. Entering the solenoid, the vortex is transformed into a magnetized state if the matching condition (4) is met.

A particular solution of Eqs. (3) has been found in Ref. [3]. It was shown that the required properties of the quadrupole block can be provided by a symmetric triplet with

which then gives

$$
\mathrm{M}=\left[\begin{array}{cc}
\cos \mu & \beta \sin \mu \\
-\beta^{-1} \sin \mu & \cos \mu
\end{array}\right],
$$

$$
\mathrm{N}=\left[\begin{array}{cc}
-\sin \mu & \beta \cos \mu \\
-\beta^{-1} \cos \mu & -\sin \mu
\end{array}\right],
$$

where $\mu$ is the free parameter.

Generally, the magnetized state created by this transformation is not round. A round beam shape inside the solenoid can be provided by matching the entrance horizontal Courant-Snyder parameters $\alpha_{x}, \beta_{x}$ with the matrix elements of the quadrupole block. For the particular solution $(5,6)$, this would require $\beta_{x}=\beta, \alpha_{x}=0$.

The reverse transformation from the magnetized into a flat beam can be done with another skew quadrupole block, described by the matrix

$$
\tilde{\mathscr{C}}=\mathscr{R}^{-1}\langle\tilde{\mathrm{M}}, \tilde{\mathrm{N}}\rangle \mathscr{R}
$$

For the example given by $(5,6)$, the vortex-to-plane transformation can be provided by the same triplet skewed by $90^{\circ}$, giving

$$
\tilde{\mathrm{M}}=\mathrm{N}, \quad \tilde{\mathrm{N}}=\mathrm{M} \text {. }
$$

Note, however, that the solution (8) for the vortex-to-plane transformation may be invalid for maps other than $(5,6)$. Indeed, a general matrix for the reverse transformation (7) can be taken as an inverse matrix of some direct transformation $\hat{\mathscr{C}}$ :

$$
\tilde{\mathscr{C}} \equiv \mathscr{R}^{-1}\langle\tilde{\mathrm{M}}, \tilde{\mathrm{N}}\rangle \mathscr{R}=\hat{\mathscr{C}}^{-1} \equiv\left(\mathscr{R}^{-1}\langle\hat{\mathrm{M}}, \hat{\mathrm{N}}\rangle \mathscr{R}\right)^{-1}=\mathscr{R}^{-1}\left\langle\hat{\mathrm{M}}^{-1}, \hat{\mathrm{N}}^{-1}\right\rangle \mathscr{R}
$$

where $\hat{\mathrm{M}}, \hat{\mathrm{N}}$ are arbitrary matrices satisfying Eq. (3), or the plane-to-vortex matrices, while the matrices $\tilde{M}, \tilde{N}$ are related to the reverse, or the vortex-to-plane, transformation. As it follows from (9), $\tilde{M}=\hat{M}^{-1}, \tilde{N}=\hat{N}^{-1}=(F \hat{M})^{-1}=-\hat{M}^{-1} F$ or

$$
\tilde{\mathrm{N}}=-\tilde{\mathrm{M}} F \text {. }
$$

The last formula describes the general solution for the vortex-to-plane mapping (7). Notice the opposite signs and the multipliers order in Eqs. (3) and (10). We have to note here that the requirement on the vortex-to-plane mapping formulated in the original paper [2] differs from our formula (10) by the order of the multipliers, which we interpret as a misprint in Ref. [2]. This generally incorrect order is also used in Refs. [3,4]. However, in these papers the discussion is limited to a particular solution $(5,6)$ where the matrices $\mathrm{N}, \mathrm{M}, \mathrm{F}$ all commute with each other; generally, though, this commutation does not take place. 
Originally, the beam adapter was proposed by Ya. Derbenev for relativistic electron cooling; he suggested to transform a naturally flat electron beam in a storage ring into a low-temperature magnetized beam inside a cooler. The reverse transformation, from a magnetized to a flat state, was later proposed as a source of a flat electron beam for linear colliders [4]. For these and possibly other applications it would be convenient to express properties of the quadrupole blocks (3) and (10) in terms of the Courant-Snyder (Twiss) parameters; this is discussed below.

\section{Betatron Functions}

\subsection{Plane-to-Vortex Transformation}

Any $2 \times 2$ linear transformation $\mathrm{T}$ can be presented in terms of the Courant-Snyder parameters (see e. g. [5]):

$$
\mathrm{T}=\left[\begin{array}{cc}
\sqrt{\frac{\beta_{2}}{\beta_{1}}}\left(\cos \mu+\alpha_{1} \sin \mu\right) & \sqrt{\beta_{1} \beta_{2}} \sin \mu \\
-\frac{1+\alpha_{1} \alpha_{2}}{\sqrt{\beta_{1} \beta_{2}}} \sin \mu+\frac{\alpha_{1}-\alpha_{2}}{\sqrt{\beta_{1} \beta_{2}}} \cos \mu & \sqrt{\frac{\beta_{1}}{\beta_{2}}}\left(\cos \mu-\alpha_{2} \sin \mu\right)
\end{array}\right] .
$$

For the plane-to-vortex transformation (1), both matrices $\mathrm{M}$ and $\mathrm{N}$ of the uncoupled quadrupole block $\langle\mathrm{M}, \mathrm{N}\rangle$ can be presented in this manner. The number of parameters forming matrix (11) is 5 , while any $2 \times 2$ symplectic matrix is determined by only 3 parameters. Thus, without any restriction, two of the parameters in (11) can be assigned to arbitrary values, which choice is a matter of convenience. We pick out $\beta_{2}=\beta, \alpha_{2}=0$ for both matrices, arbitrary referring to a round shape of the vortex state (more see below). After that, the both matrices are fully described by the input Courant-Snyder parameters $\alpha_{1}, \beta_{1}$ and the phase advance $\mu$. To distinguish between the parameters for the two matrices, the subscript $\xi$ is used for the matrix $\mathrm{M}$ parameters and the subscript $\eta$ for the matrix $\mathrm{N}$, implying that $\xi$ and $\eta$ are the natural axes of the diagonal block $\langle\mathrm{M}, \mathrm{N}\rangle$. Then, the plane-to-vortex conditions (3) result in very simple relations for these two sets of the Courant-Snyder parameters. Namely, it follows that the requirements (3) are satisfied if the entrance betatron functions of $\mathrm{M}$ and $\mathrm{N}$ transformations are equal, while their phase advances differ by $90^{\circ}$ :

$$
\beta_{1 \eta}=\beta_{1 \xi}, \alpha_{1 \eta}=\alpha_{1 \xi}, \mu_{\eta}=\mu_{\xi}-\pi / 2 .
$$

The conditions (12) and (3) are equivalent. When they are satisfied, any ribbon-like, horizontally oriented, beam $\left(\varepsilon_{y} / \varepsilon_{x}<<1\right)$ transforms into a vortex at its exit.

The entering flat beam can be presented by its bounding phase ellipse as

$$
x=\sqrt{2 J \beta_{x}} \cos \psi, \quad x^{\prime}=-\sqrt{2 J / \beta_{x}}\left(\alpha_{x} \cos \psi+\sin \psi\right),
$$

where $J$ is a bounding action. Generally, this $x-x$ ' ellipse is transformed into a vortex whose $x-y$ cross-section is also elliptical, and the axes are turned by a certain angle. In 
particular, this vortex ellipse can be a circle. It is straightforward to see that the vortex is round when the entering flat beam is matched with the adapter, i. e.

$$
\beta_{x}=\beta_{1 \xi}, \alpha_{x}=\alpha_{1 \xi} .
$$

In this case, the vortex radius

$$
r=\sqrt{J \beta}
$$

A simplicity of relations $(14,15)$ is reached due to a particular choice made above for the exit Courant-Snyder parameters $\beta_{2}=\beta, \alpha_{2}=0$ for the both matrices $\mathrm{M}$ and $\mathrm{N}$.

\subsection{Vortex-to-Plane Transformation}

Similar analysis can be performed for the reverse, the vortex-to-plane transformation. In this case, the same reasoning leads to a choice of $\widetilde{\beta}_{1}=\beta, \quad \widetilde{\alpha}_{1}=0$; then the vortex-toplane conditions (10) are equivalent to a requirement on the exit Courant-Snyder parameters

$$
\tilde{\beta}_{2 \eta}=\tilde{\beta}_{2 \xi}, \quad \tilde{\alpha}_{2 \eta}=\tilde{\alpha}_{2 \xi}, \quad \tilde{\mu}_{\eta}=\tilde{\mu}_{\xi}+\pi / 2 .
$$

When the conditions (10) or (16) are satisfied, any magnetized beam exiting the matched solenoid (4) transforms into a ribbon-like $x$-oriented state after the skew block. A round beam inside the solenoid with the radius (15) results in a flat beam with the phase ellipse (13) and $\beta_{x}=\widetilde{\beta}_{2}, \alpha_{x}=\tilde{\alpha}_{2}$.

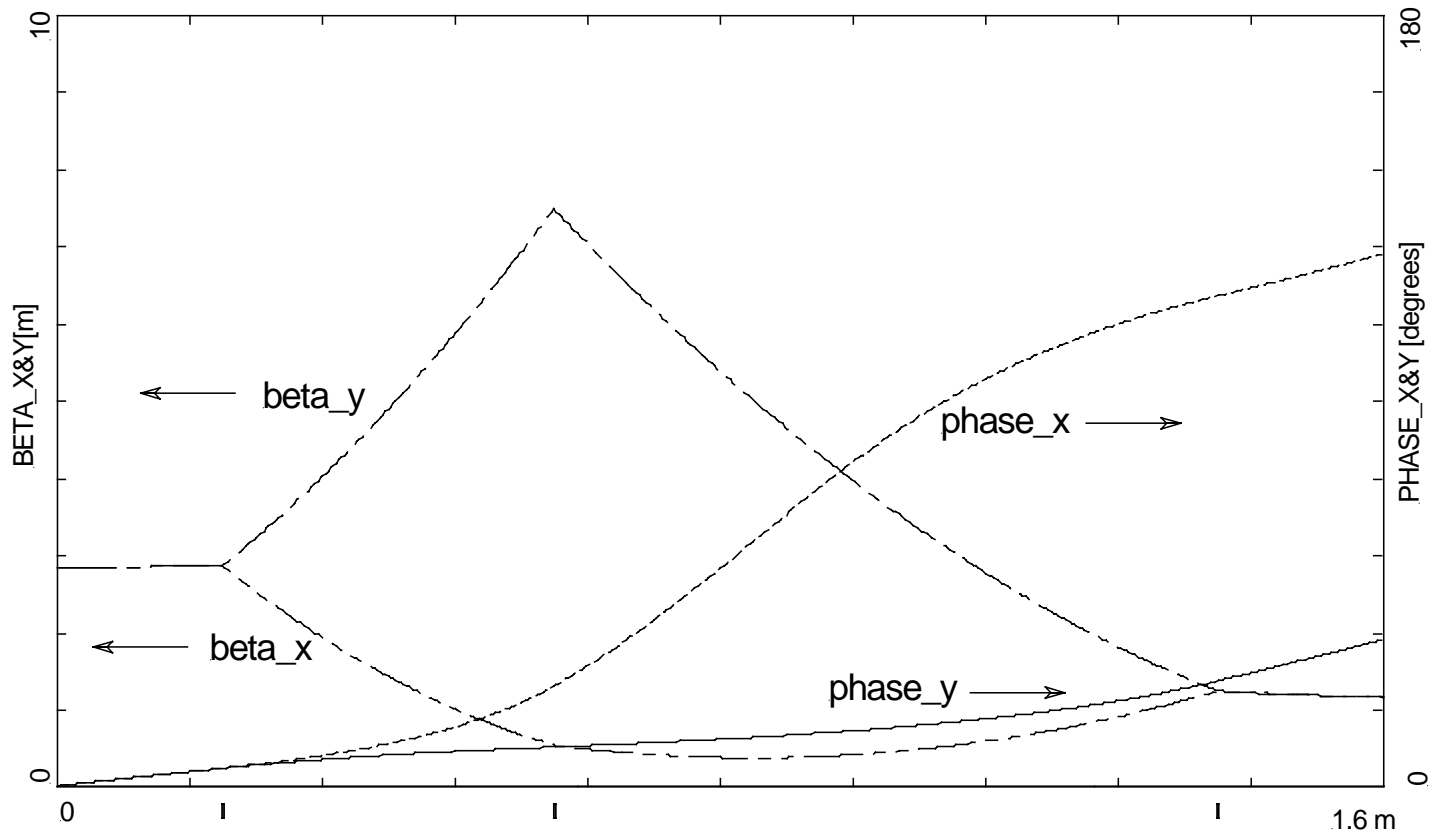

Figure 1: Courant-Snyder parameters of a non-skewed asymmetric triplet. In this example the triplet parameters are as follows: $l_{1}=20 \mathrm{~cm}, f_{1}=66.1 \mathrm{~cm}, l_{2}=40 \mathrm{~cm}, f_{2}=$ $57.9 \mathrm{~cm}, l_{3}=80 \mathrm{~cm}, f_{3}=66.8 \mathrm{~cm}$, and $l_{4}=20 \mathrm{~cm}$, counting the drifts $l_{i}$ and the focal lengths $f_{i}$ from left to right as they are encountered by the beam. 
Figures 1 and 2, obtained with the OptiM code [5], show an example of Courant-Snyder parameters, phases, and beam envelopes for the vortex-to-plane transformation. The beam energy in this example is $p c=17 \mathrm{MeV}$, the cathode radius is $4 \mathrm{~mm}$, and the magnetic field at the cathode is $400 \mathrm{G}$, which yields $\beta=2.83 \mathrm{~m}$. The three conditions (16) are provided by a proper choice of the quadrupole strength of an asymmetric triplet with the drift spaces kept fixed.

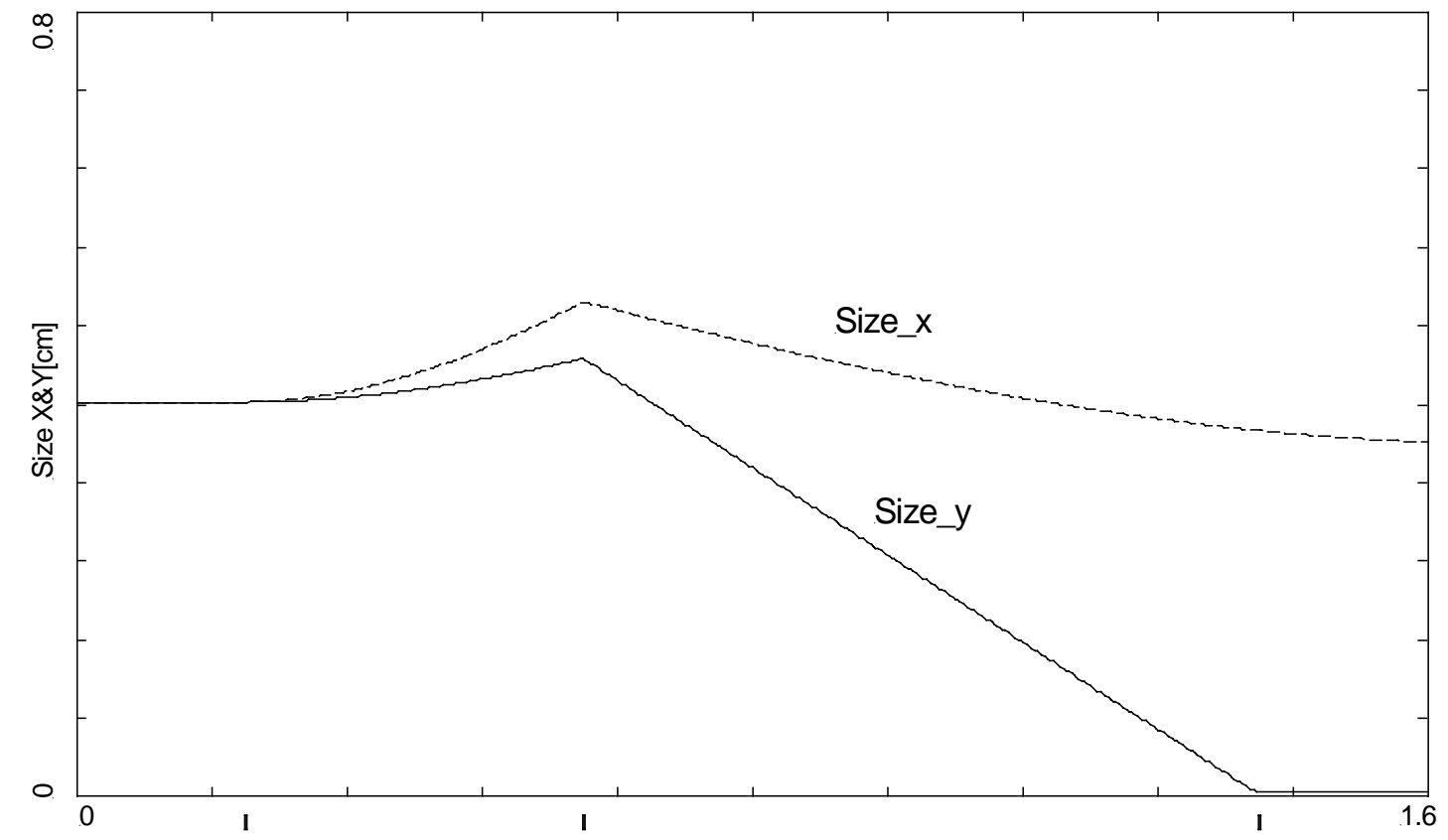

Figure 2: The horizontal and vertical beam sizes resulted from the vortex-to-plane transformation of Fig. 1.

\section{Canonical Emittances for the Adapter}

In this section, the adapter is considered in terms of a 4D phase space transformation, so that both the horizontal and vertical degrees of freedom of the "flat" beam are assumed to be excited. Similarly, the resulting Larmor rotation inside the solenoid is not neglected or even assumed to be small. The terms for the transformations, such as plane-tomagnetized, vortex-to-plane, etc., are continued to be used here, although without assumptions that the incoming beam is necessarily very "flat", or the beam inside the solenoid travels exactly along the magnetic field lines.

The particle state at any stage of the transformation can be presented by the 4D phase space vector

$$
\mathbf{x}=\left(\begin{array}{c}
x \\
p_{x} \\
y \\
p_{y}
\end{array}\right)
$$


with $\left(x, p_{x}\right)$ and $\left(y, p_{y}\right)$ as canonically conjugated variables for the horizontal and vertical degrees of freedom. Both direct (plane-to-magnetized) and reversed (magnetizedto-plane) transformations can be presented by matrices acting on vectors of the phase space (17). Both the transformations couple the horizontal and vertical degrees of freedom; therefore, such matrices look as general symplectic $4 \times 4$ matrices. However, one can choose such canonical variables that the related transformation matrices are blockdiagonal. These so-called normal coordinates, described in Ref. [6], are just the usual laboratory coordinates (17) before the direct skew-block (1) and/or after the reverse block (7), and they are the drift-cyclotron coordinates inside the solenoid. A canonical transformation from the usual coordinates to the drift-cyclotron coordinates can be described as follows.

For a given point $\mathbf{x}$ in the 4D phase space, the transverse kinematic momentum $\vec{k}$ and the position $\vec{d}$ of the Larmor center are expressed as

$$
\begin{aligned}
\vec{d} & =\vec{r}-\vec{\rho}=\frac{1}{2} \vec{r}-\frac{c}{e} \frac{\vec{p} \times \vec{B}}{B^{2}} \\
\vec{k} & =\vec{p}+\frac{e}{2 c} \vec{B} \times \vec{r}
\end{aligned}
$$

where $\vec{r}=(x, y), \vec{p}=\left(p_{x}, p_{y}\right), B$ is the uniform longitudinal magnetic field value, and the vector $\vec{\rho}=c \vec{k} \times \vec{B} /\left(e B^{2}\right)$ describes a position of the rotating particle relative to its Larmor center. The relationships (18) can be considered as transformation from the canonical pairs $\vec{r}, \vec{p}$ to the new variables $\vec{d}$ and $\vec{k}$. An amazing feature of this transformation is that the Poisson brackets

$$
\{f, g\} \equiv \sum_{i=x, y}\left(\frac{\partial f}{\partial p_{i}} \frac{\partial g}{\partial r_{i}}-\frac{\partial g}{\partial p_{i}} \frac{\partial f}{\partial r_{i}}\right)
$$

between any of $\vec{d}$ and $\vec{k}$ components are equal to zero, while

$$
\left\{d_{x}, d_{y}\right\}=\frac{c}{e B}, \quad\left\{k_{x}, k_{y}\right\}=-\frac{e B}{c} .
$$

Therefore, the normalized variables

$$
\left(\begin{array}{l}
\xi_{1} \\
\xi_{2}
\end{array}\right)=\sqrt{\frac{e B}{c}}\left(\frac{d_{x}}{d_{y}}\right),\left(\begin{array}{l}
\kappa_{1} \\
\kappa_{2}
\end{array}\right)=\sqrt{\frac{c}{e B}}\left(\frac{k_{y}}{k_{x}}\right)
$$

compose new canonical pairs (compare with Ref. [7], p. 432), referred in Ref. [5] as the cyclotron and the drift variables correspondingly. The canonical transformation from the initial variables (17) to the drift-cyclotron pairs (21) can be presented as

$$
\varsigma \equiv\left(\begin{array}{c}
\xi_{1} \\
\xi_{2} \\
\kappa_{1} \\
\kappa_{2}
\end{array}\right)=\mathscr{B}\left(\begin{array}{c}
x \\
p_{x} \\
y \\
p_{y}
\end{array}\right) \equiv \mathscr{B} \mathbf{x}
$$

with a symplectic $4 \times 4$ matrix $\mathscr{B}$, which can be composed using Eqs. (18) and (21). The skew block (1) transforms the incoming horizontal beam into a vortex. A vertical beam 
would be transformed by this block into a vortex as well, but with an opposite twist. Then, the solenoidal fringe field cancels this rotation in the first case and doubles it in the second. Thus, the horizontal motion of the incoming beam is transformed into a pure drift, while the vertical motion is transformed into the pure cyclotron motion, or the Larmor rotation around the axis of the solenoid. Thus, the drift-cyclotron coordinates (21) are the normal coordinates inside the matched solenoid of the adapter, i. e. the plane-tomagnetized mapping is described by the $4 \times 4$ block-diagonal symplectic matrix $\mathscr{A}$, when presented in terms of the usual entrance coordinates (17) and the drift-cyclotron exit coordinates (22):

$$
\varsigma=\mathscr{A} \mathbf{x}, \quad \mathscr{A}=\left(\begin{array}{cc}
(\mathrm{DX}) & 0 \\
0 & (\mathrm{CY})
\end{array}\right) .
$$

with $2 \times 2$ matrices (DX) ( $x$-to-drift) and (CY) ( $y$-to-cyclotron). The symplecticity condition for the matrices like (23) reduces to a requirement for the partial determinants

$$
\operatorname{det}(D X)=\operatorname{det}(C Y)=1 \text {. }
$$

A consequence of the presentation $(23,24)$ is that the horizontal and vertical emittances are restored as the drift and the cyclotron emittances correspondingly:

$$
\begin{aligned}
& \varepsilon_{x}^{2} \equiv\left\langle x^{2}\right\rangle\left\langle\theta_{x}^{2}\right\rangle-\left\langle x \theta_{x}\right\rangle^{2}=\varepsilon_{d}^{2} \equiv\left(\left\langle\xi_{1}^{2}\right\rangle\left\langle\xi_{2}^{2}\right\rangle-\left\langle\xi_{1} \xi_{2}\right\rangle^{2}\right) / p_{0}^{2} \equiv \frac{4}{\beta^{2}}\left(\left\langle d_{x}^{2}\right\rangle\left\langle d_{y}^{2}\right\rangle-\left\langle d_{x} d_{y}\right\rangle^{2}\right) \\
& \varepsilon_{y}^{2} \equiv\left\langle y^{2}\right\rangle\left\langle\theta_{y}^{2}\right\rangle-\left\langle y \theta_{y}\right\rangle^{2}=\varepsilon_{c}^{2} \equiv\left(\left\langle\kappa_{1}^{2}\right\rangle\left\langle\kappa_{2}^{2}\right\rangle-\left\langle\kappa_{1} \kappa_{2}\right\rangle^{2}\right) / p_{0}^{2} \equiv \frac{\beta^{2}}{4}\left(\left\langle\vartheta_{x}^{2}\right\rangle\left\langle\vartheta_{y}^{2}\right\rangle-\left\langle\vartheta_{x} \vartheta_{y}\right\rangle^{2}\right)
\end{aligned}
$$

where $\theta_{x, y}, \vartheta_{x, y}$ are the beam angles at the entrance of the skew block and in the solenoid respectfully, the brackets $\langle\ldots\rangle$ denote an ensemble averaging, and the parameter $\beta$ is defined in Eq. (4). For a particular case of the round beam inside the solenoid with $\left\langle d_{x}^{2}\right\rangle=\left\langle d_{y}^{2}\right\rangle=\left\langle d^{2}\right\rangle / 2,\left\langle d_{x} d_{y}\right\rangle=0$, and the similar angle relations, it yields

$$
\varepsilon_{x}=\frac{\left\langle d^{2}\right\rangle}{\beta}, \quad \varepsilon_{y}=\frac{\beta\left\langle\vartheta^{2}\right\rangle}{4} \text {. }
$$

The reverse, magnetized-to-plane transformation $\tilde{\mathscr{A}}$ can be presented in a similar way:

$$
\mathbf{x}=\tilde{A} s, \quad \tilde{A}=\left(\begin{array}{cc}
(\mathrm{XD}) & 0 \\
0 & (\mathrm{YC})
\end{array}\right)
$$

with, again, symplectic $2 \times 2$ matrices $(X D)$ and $(Y C)$. Obviously, relations of the emittance preservation (25) are valid for the reverse transformation as well.

\section{Preparation of Flat Beams with Desirable Density Distribution}

The adapting transformations $(23,27)$ map the horizontal phase space onto the transverse cross-section and reverse. In particular, the magnetized-to-flat transformation of a beam from a magnetized cathode maps a shape of the cathode onto the horizontal phase space of the outgoing flat beam. Changing the cathode shape, the surface density distribution can be modified in arbitrary way, so any distribution function can be prepared. 
The magnetized-to-flat transformation was suggested to be used for preparation of flat beams for linear colliders [4]. Beams with a homogeneous (step-like) surface density might be of a particular interest here, corresponding to a maximal luminosity for a given beam-beam parameter. Thus, a question appears about a shape of the magnetized cathode corresponding to a homogeneous density of the flat beam at the interaction point. An answer can be found from very general considerations. If the horizontal phase space of the beam is limited by a rectangular $|x|<x_{m}, \quad\left|x^{\prime}\right|<x_{m}^{\prime}$, its density is independent of the horizontal offset. Due to linearity of the mapping, the reverse image of the $x$ - $x$ ' rectangular is a parallelogram in the $x-y$ plane. Thus, the cathodes of the parallelogram shape can be used for a preparation of the homogeneous flat beams. In particular, homogeneous (step-like) beam surface density yields after a proper transformation of a magnetized beam shaped as an upright diamond. For instance, this diamond shape could be a square.

\section{Summary}

In this paper, a general requirement on the recently suggested magnetized-to-flat mapping was discussed. The properties of the involved quadrupole blocks were considered in terms of the Courant-Snyder parameters for both the direct and reverse adapting transformations. Emittance preservation was formulated for both the emittances involved. A possibility for preparation of arbitrary density distribution of the flat beam was pointed out.

The authors are thankful to Yaroslav Derbenev, Don Edwards, Helen Edwards, and Valeri Lebedev for numerous fruitful discussions.

\section{References}

[1] Ya. Derbenev, “Adapting Optics for High Energy Electron Cooling” UM HE 98-04, University of Michigan, 1998.

[2] Ya. Derbenev, “Advanced Optical Concepts for Electron Cooling”, NIM-A, 441, p. 223 (2000).

[3] A. Burov and V. Danilov, "An Insertion to Eliminate Horizontal Temperature of High Energy Electron Beam”, FERMILAB-TM-2043, 1998.

[4] R. Brinkmann, Ya. Derbenev and K. Flöttmann, “A Flat Beam Electron Source for Linear Colliders", TESLA 99-09, DESY, 1999.

[5] V. A. Lebedev, at http://host.sybercom.net/users/ldbs; this site is a home of LDBS Co. developing the OptiM beam optics code. 
[6] A. Burov, S. Nagaitsev, A. Shemyakin and Ya. Derbenev, "Optical Principles of Beam Transport for Relativistic Electron Cooling”, FERMILAB-Pub-00/100-T (2000), also submitted to PRST-AB.

[7] H. Goldstein, "Classical Mechanics", Addison-Wesley, Mass., Second Edition, 1980 